Such strong diffusional anisotropy is not observed in other deep-mantle minerals, and it introduces some ambiguity in modelling the rheology of $\mathrm{D}^{\prime \prime}$. Post-perovskite could be either stronger or weaker than perovskite depending on how it deforms - whether by 'diffusion creep', in which case the slow direction dominates, or by 'dislocation creep', in which the faster direction may control the rate of deformation. Diffusion creep cannot easily explain the anisotropic behaviour of seismic waves observed $^{2}$ in $\mathrm{D}^{\prime \prime}$. Thus, it seems likely that dislocation creep is the main mode of deformation, and that the transition to post-perovskite results in a substantial reduction in resistance to flow. This interpretation is broadly consistent with geophysical estimates of the mantle's viscosity profile ${ }^{6}$, but suggests that $\mathrm{D}^{\prime \prime}$ is much weaker than previously thought.

Ammann and colleagues ${ }^{1}$ also propose a mechanism for generating the sharp reflection of seismic waves that is observed at the upper boundary of $\mathrm{D}^{\prime \prime}$. Although the mineralogical transformation from perovskite to post-perovskite may be gradual ${ }^{7}$, an abrupt transition in rheology might occur when a critical fraction of the new mineral is produced. A shift from perovskite-dominated diffusion creep to post-perovskite-dominated dislocation creep may lead to a shift from an isotropic mineral fabric above $\mathrm{D}^{\prime \prime}$ to a textured one in which the mineral grains have a high degree of crystallographic alignment. The increase in the speed with which waves travel in the textured material may be sufficient to reflect seismic waves at this rheological transition.

Clearly, much work remains to be done to provide a more complete picture of the rheology of deep-mantle materials. Deformation processes in minerals with strong diffusion anisotropy need to be better understood, as do the effects of chemical impurities. Ultimately, technical developments should permit wellcontrolled diffusion and deformation experiments at the conditions relevant to $\mathrm{D}^{\prime \prime}$. But for now, sophisticated computations such as those performed by Ammann and colleagues are leading the way.

James A. Van Orman is in the Department of Geological Sciences, Case Western Reserve University, Cleveland, Ohio 44106, USA.

e-mail: james.vanorman@case.edu

Ammann, M. W., Brodholt, J. P., Wookey, J. \& Dobson, D. P. Nature 465, 462-465 (2010).

2. Lay, T., Williams, Q. \& Garnero, E. J. Nature 392, 461-468 (1998).

3. Karato, S. \& Weidner, D. J. Elements 4, 191-196 (2008).

4. Murakami, M., Hirose, K., Kawamura, K., Sata, N. \& Ohishi, Y. Science 304, 855-858 (2004)

5. Wentzcovitch, R. \& Stixrude, L. (eds) Rev. Miner. Geochem. 71(2010).

6. Mitrovica, J. X. \& Forte, A. M. Earth Planet. Sci. Lett. 225, 177-189 (2004)

7. Catalli, K., Shim, S.-H. \& Prakapenka, V. Nature 462, 782-785 (2009)

\title{
CANCER
}

\section{A wolf in wolf's clothing}

\author{
Douglas R. Green
}

\section{Paradoxically, the CD95 receptor, a potent inducer of apoptotic cell death, is expressed on most tumour cells. Surprisingly, it turns out to be an important promoter of various cancers.}

For more than two decades, CD95 - also known as Fas and APO-1 - has been considered a killer, the capo di tutti capi of death receptors in the tumour-necrosis-factor receptor family. Interaction of this cell-surface receptor with its ligand, CD95L, or with activating antibodies induces rapid apoptotic death in many cell types, and injection of such ligands or antibodies into animals results in liver destruction and death. Moreover, deletion or mutation of CD95 causes an immunerelated disease due to loss of apoptosis. In many cells, even when apoptosis is blocked, CD95 induces necrotic cell death through a different signalling pathway. So the idea that CD95 actually promotes cancer - which is generally characterized by decreased rather than increased cell death - seems ridiculous. But on page 492 of this issue, Chen and colleagues ${ }^{1}$ provide overwhelming evidence that this is indeed the case, a finding that is tantamount to a paradigm shift.

The authors use various human tumourcell lines, primary cancer cell lines and mouse models of spontaneous and damage-induced cancer to show that reducing or abolishing CD95 compromises tumour growth without causing cell death. Furthermore, they find that tumour-derived CD95L is necessary for tumour promotion, presumably acting in a cell-intrinsic (autocrine) way. Their conclusion, that CD95L-CD95 signalling is generally important for the generation and maintenance of cancers, is therefore compelling.

Hints that CD95 has non-apoptotic functions have surfaced repeatedly in the past. (Sometimes, however, a viewpoint becomes so ingrained in our collective scientific consciousness that alternatives go unheeded.) For example, previous work has shown that activation of CD95 has a co-stimulatory effect with antigen-receptor signalling in T lymphocytes,

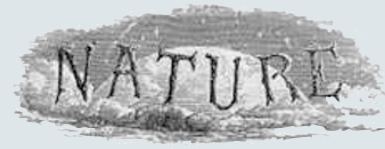

\section{YEARS AGO}

Since Morrison and Cocconi published the suggestion that there might be advanced societies elsewhere in the Galaxy ... beaming transmissions to us on a frequency of $1,420 \mathrm{Mc} . / \mathrm{s}$, Drake has described equipment under construction to look for such transmissions ... Would not this other more advanced society ... be doing what we ourselves are now discussing and are on the point of doing, probably during this century, namely, sending probes to nearby stars ... For this reason we might better devote our efforts to scrutinizing our solar system for signs of probes sent here by our more advanced neighbours.

From Nature 28 May 1960

\section{YEARS AGO}

In the Cairo Scientific Journal for February Mr. Harold Sheridan gives an account of that curious musical instrument, the rabába, which was introduced into Europe by the Crusaders, and, with a slight modification of the original name, is now known as the rebeck. It has certainly been evolved from the one-string lyre of the early monuments, the single string twanged with the finger developing into the present double-stringed instrument played with a rude bow and provided with a body. Even in its present state it is a most primitive instrument, made up in the rudest way out of a long iron nail, a cocoa-nut, a few strands of horse-hair (that of the living animal being most in request), a piece of fish-skin, and sundry pieces of wood. The last are coarsely glued together, and the body is made of half the cocoa-nut, over which a piece of moist skin that of the Nilotic fish known as the bayad - is tied tightly until it dries. The tone is regulated by incisions made in the body ... The rabába is thus of considerable interest as marking an early stage in the evolution of the modern violin.

From Nature 26 May 1910. 
a

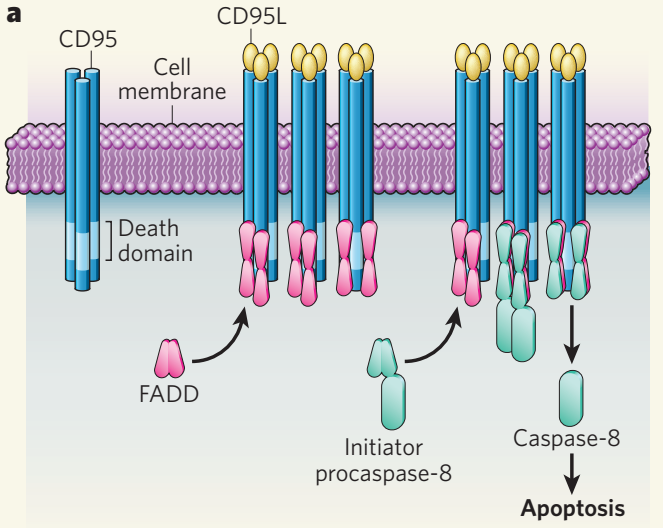

b

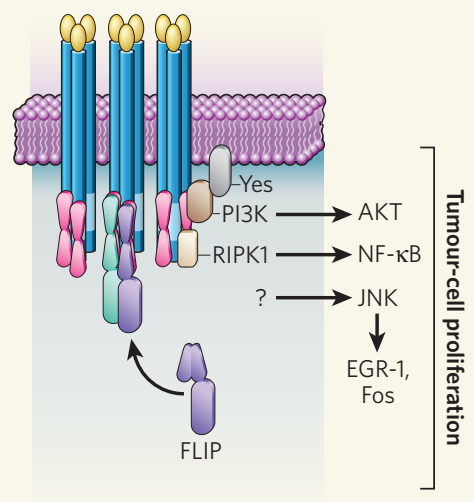

Figure 1 | CD95 signalling for apoptosis, and for tissue growth and tumour promotion. On binding to its ligand, CD95L, CD95 generates different signals, depending on the cell type and other conditions. a, For apoptosis, the adaptor protein FADD must be recruited to the exposed death domain of the activated receptor. FADD, in turn, recruits the initiator procaspase-8, which cleaves itself to form the active caspase-8 - an enzyme that promotes apoptosis. b. When cells express FLIP, apoptosis does not occur and, instead, other cell-type-dependent signalling events become manifest. These include activation of JNK by an unknown mechanism (leading to expression of EGR-1 and Fos), of NF- $\mathrm{kB}$ by a scaffold complex involving RIPK1, and of PI3 kinase (PI3K), mediated by Yes and p85 (not shown), and its downstream effector, AKT. The tumour-promoting functions of CD95, which Chen et al. describe, may involve some or all of these non-apoptotic activities.

and that neurons in which CD95 is triggered display neurite outgrowth ${ }^{2}$. In addition, Chen and colleagues ${ }^{1}$ show that ablation of CD95 in the liver slows compensatory proliferation of liver cells following partial liver resection (hepatectomy).

So how does CD95 promote growth? This receptor's only known ligand, CD95L, can be either membrane bound or cleaved to a soluble form. The membrane-bound form is required for CD95L-induced apoptosis, and animals that can express only the soluble form develop immune-system abnormalities more rapidly than do CD95L-deficient mice ${ }^{3}$. Remarkably, these animals also develop hepatic sarcoma, a form of cancer rarely seen in animals lacking CD95 or CD95L. The idea that the soluble form of CD95L might be responsible for CD95 signals that promote tumour formation is therefore attractive. Indeed, patients with cancer often have high levels of soluble CD95L in their blood.

Inflammation is an important factor in promoting cancer ${ }^{4}$. So one idea is that CD95 aids tumour growth by inducing inflammation. Expression of CD95L in abnormal locations such as pancreatic islet cells or transplanted tissues can induce a dramatic infiltration of white blood cells ${ }^{5}$ - a hallmark of inflammation. Against this idea, however, Chen et al. report that CD95L promotes tumour growth by mechanisms within the tumour, with no profound differences in inflammation between tumours expressing CD95 and those that do not express it. Furthermore, earlier work ${ }^{6}$ has shown that, unlike membrane-bound CD95L, soluble
CD95L does not promote inflammation.

Activation of death receptors, including CD95, triggers several signalling pathways besides that leading to apoptosis (Fig. 1). But which of these signals might promote tumour growth? It has been proposed that activation of the transcription factor NF- $\kappa \mathrm{B}$ (probably through the RIPK1 enzyme, which is recruited to the death-induced signalling complex, DISC) might account for the growth-promoting effects of CD95 activation ${ }^{3}$. But this seems unlikely, because although CD95 promotes damage-induced hepatocellular carcinoma ${ }^{1}$, this form of cancer is exacerbated in mice that lack the canonical NF- $\kappa \mathrm{B}$ signalling pathway in their livers ${ }^{4}$.

Alternatively, Chen et al. ${ }^{1}$ suggest a role for the enzyme JNK, and increased expression of EGR-1 and Fos transcription factors downstream of it. They show that chemical inhibition of JNK retards the growth of CD95expressing tumour cells. Moreover, CD95 activation in liver cells undergoing proliferation after partial hepatectomy (a situation that may be mechanistically similar to tumour initiation) profoundly increases levels of EGR-1 and Fos.

Unfortunately, we know almost nothing about how CD95 might activate JNK. Soluble CD95L does not trigger JNK activation ${ }^{7}$, but a deeper problem is that, in the absence of CD95, JNK can be activated in many other ways. Indeed, several other related proteins — such as tumour-necrosis factor - readily induce JNK, and it is difficult to see why CD95 would be needed for this signal to promote cancer. In other words, what is so special about CD95?
Apoptosis in response to CD95 activity is controlled by proteins that associate with the intracellular death domain of this receptor, such as the adaptor protein FADD, leading to the recruitment of DISC and activation of the enzyme caspase-8 (Fig. 1). FLIP - a caspaselike molecule without proteolytic activity - alters this signalling in favour of survival. Presumably, this is how tumour cells avoid death signalling by CD95. But there is a hint that this is not the whole story.

Animals with defective CD95 expression exhibit delayed liver-cell proliferation and liver regeneration after partial hepatectomy, whereas animals with a mutation in CD95 known as $l p r^{c g}$, which disrupts the structure of the death domain, show normal regeneration $^{8}$. Remarkably, when $1 \mathrm{pr}^{\mathrm{cg}}$-mutant mice were provided with a wild-type haematopoietic system (to prevent the immune-system disease), they developed liver tumours ${ }^{9}$. It seems, therefore, that the tumour-promoting signal that CD95 generates - perhaps normally offset by CD95-induced apoptosis - persists in this mutant.

Such a signal might come about through the recruitment and activation of the enzyme PI3 kinase. In glioblastoma cells, activated CD95 recruits both the Src-family kinase Yes and the p85 subunit of PI3 kinase, leading to PI3-kinase activation ${ }^{10}$. Yes and p85 interact with the membrane-proximal region of the CD95 death domain, which is likely to remain intact in the $\mathrm{lpr}^{\mathrm{cg}}$ mutant, although this has not been tested. Thus, CD95 activation may promote the activity of the signalling molecule AKT, downstream of PI3 kinase, to induce cell proliferation.

Whatever the mechanism, a general role for autocrine CD95 signalling in promoting cancer is a stunning revelation that goes against many of the prevailing ideas of what this receptor does. There are obvious practical consequences as we explore the benefits of blocking CD95L-CD95 interactions in cancer therapy. But perhaps most of all, as we confirm the generality of this process in cancers, there is our realization that this 'wolf' - this potentially deadly tumour-promoting mechanism - has been there all along, disguised as a mechanism of cell death.

Douglas R. Green is in the Department of Immunology, St Jude Children's Research Hospital, Memphis, Tennessee 38105, USA. e-mail: douglas.green@stjude.org

1. Chen, Letal Nature 465,492-496(2010).

2. Peter, M. E. et al. Cell 129, 447-450 (2007).

3. O'Reilly, L. A. et al. Nature 461, 659-663 (2009).

4. Grivennikov, S. I., Greten, F. R. \& Karin, M. Cell 140, 883-899 (2010).

5. Kang, S.-M. et al. Nature Med. 3, 738-743 (1997).

6. Shudo, K. et al. Eur. J. Immunol. 31, 2504-2511 (2001).

7. Villunger, A., Huang, D. C. S., Holler, N., Tschopp, J. \& Strasser, A. J. Immunol. 165, 1337-1343 (2000).

8. Desbarats, J. \& Newell, M. K. Nature Med. 6, 920-923 (2000).

9. Park, S.-M. et al. Apoptosis 13, 41-51 (2008)

10. Sancho-Martinez, I. \& Martin-Villalba, A. Cell Cycle 8, 838-842 (2009). 